JOURNAL OF

FUNCTION SPACES AND APPLICATIONS

Volume 7, Number 2 (2009), 105-120
(C) 2009, Scientific Horizon

http://www.jfsa.net

\title{
A Korovkin theorem in multivariate modular function spaces
}

\author{
Carlo Bardaro and Ilaria Mantellini \\ (Communicated by Jürgen Appell)
}

2000 Mathematics Subject Classification. 41A35, 47G10, 46E30.

Keywords and phrases. Modular function spaces, linear operators, Korovkin theorem, moments.

\footnotetext{
Abstract. In this paper a modular version of the classical Korovkin theorem in multivariate modular function spaces is obtained and applications to some multivariate discrete and integral operators, acting in Orlicz spaces, are given.
}

\section{Introduction}

The class of modular function spaces was introduced, for the first time, by H. Nakano [29] and then extensively studied by J. Musielak [27] who developed a theory of approximation in this general frame for classes of linear and nonlinear operators $([28])$. An abstract approach to the theory of approximation was given in its definite form in [4]. This book represents the first attempt at a comprehensive treatment of approximation theory in modular spaces for nets of nonlinear operators. The interest in working in such general spaces is mainly to ensure an unifying approach which includes, by a unique method, several results in various functional spaces. Indeed modular function spaces include $L^{p}$-spaces, Orlicz spaces, Musielak-Orlicz 
spaces, the spaces of functions with bounded variation, Orlicz-Sobolev spaces and more ([27], [23], [4]).

One of the most interesting results in classical approximation theory is certainly given by the Korovkin theorem ([21], [22], [7]). The classical Korovkin theorem states the uniform convergence in $C([a, b])$, the space of the continuous real functions defined on $[a, b]$, of a sequence of positive linear operators, by stating the convergence only on three test functions $\left\{1, x, x^{2}\right\}$. The work of Korovkin was inspired by the Bernstein proof of the Weierstrass theorem ([6]). Here the author established the uniform convergence of the Bernstein polynomials of the function $f$ by stating it only on the functions $\left\{1, x, x^{2}\right\}$. There is also a trigonometric version of the Korovkin theorem, using the test functions $\{1, \cos x, \sin x\}$, see [22], [9]. Later on several extensions of the Korovkin theorem were obtained in various settings. We quote here the books [12], [24], [13], [1] and the extensive survey [16], which contains a wide list of references. Other interesting generalizations were obtained in [18], [31].

Recently, versions of the Korovkin theorem were obtained in different functional spaces, namely $L^{p}$-spaces or abstract Lebesgue spaces (see, e.g., [5], [19], [26], [20], [14],[15], [8], [30], [32]. For more references on this topic see [1], Appendix D).

In [3], we obtained an extension of the Korovkin theorem in the abstract setting of the modular function spaces for real functions defined on a compact interval $[a, b]$, using the classical test set $\left\{1, x, x^{2}\right\}$ and its elementary properties. In the present paper, we give a modular version of the Korovkin theorem in multivariate modular function spaces. We start with a generalized version of the Korovkin theorem for functions defined on open precompact sets in a Hausdorff locally compact topological space, provided with a regular measure defined on the Borel sets, in which a general test set is used satisfying suitable assumptions. This general approach is quite different from the classical one. Here we use a modification, suitable for modular function spaces, of a technique employed in [24] (see also [31]). Note that for certain function spaces, as for example $L^{p}$-spaces, in general it is not possible to get the convergence in $L^{p}$ of a sequence of positive linear operators for all the $L^{P}$ functions, but it is necessary to consider suitable subspaces, depending on the form of the operators involved. Given a finite class of functions $\left\{e_{i}\right\}$ and a sequence $\mathbf{T}=\left(T_{n}\right)$ of positive linear operators such that $\left(T_{n} e_{i}\right)$ converges to $e_{i}$, with respect to the Luxemburg norm in the modular space, we determine a subspace $X_{\mathbf{T}}$ such that $\left(T_{n}\right)$ converges with respect to the modular topology on every function of this subspace. Key tools for this result are a density property of the space of the continuous functions in the modular space (see [25]) and a kind of "approximate" modular continuity assumption on the sequence $\left(T_{n}\right)_{n \in \mathbb{N}}$ over the class. In 
particular we obtain, as a special case, a version of the Korovkin theorem in $L^{p}$ spaces and in Orlicz or Musielak-Orlicz spaces. In Section 4, we apply our general theory to some kind of discrete operators acting on multivariate functions defined on nonempty bounded subsets of $\mathbb{R}^{n}$. Then in Section 5 we consider the case of Mellin type integral operators (see [10]) for one dimensional Mellin convolution operators. Our result can be applied to various classical operators like multivariate Bernstein operators ([24]) and multivariate moment operators ([11] and [17]).

\section{Notations and definitions}

Let $A$ be a nonempty open set in a Hausdorff locally compact topological space $H$ provided with a regular measure $\mu$ defined on the Borel sets of $H$. We will assume that $\bar{A}$ is compact. We will denote by $X(A)$ the space of all real-valued Borel measurable functions $f: A \rightarrow \mathbb{R}$ provided with equality $\mu$-a.e., by $C(A)$ the space of all continuous and bounded real functions defined on $A$ and by $C_{u}(A)$ the subset of $C(A)$ whose elements have a continuous extension to $\bar{A}$. A functional $\varrho: X(A) \rightarrow \overline{\mathbb{R}_{0}^{+}}$is said to be a modular on $X(A)$ if

i) $\varrho[f]=0 \Leftrightarrow f=0$, a.e. in $\mathrm{A}$,

ii) $\varrho[-f]=\varrho[f]$, for every $f \in X(A)$,

iii) $\varrho[\alpha f+\beta g] \leq \varrho[f]+\varrho[g]$, for every $f, g \in X(A), \alpha, \beta \geq 0, \alpha+\beta=1$.

We will say that a modular $\varrho$ is Q-quasi convex if there is constant $Q \geq 1$ such that

$$
\varrho[\alpha f+\beta g] \leq Q \alpha \varrho[Q f]+Q \beta \varrho[Q g],
$$

for every $f, g \in X(A), \alpha, \beta \geq 0, \alpha+\beta=1$. If $Q=1$ we will say that $\varrho$ is convex. By means of the functional $\varrho$, we introduce the vector subspace of $X(A)$, denoted by $L^{\varrho}(A)$, defined by

$$
L^{\varrho}(A)=\left\{f \in X(A): \lim _{\lambda \rightarrow 0^{+}} \varrho[\lambda f]=0\right\} .
$$

The subspace $L^{\varrho}(A)$ is called the modular space generated by $\varrho$. It is easy to see that when $\varrho$ is $Q$-quasi-convex we have the following characterization of the modular space $L^{\varrho}(A)$ :

$$
L^{\varrho}(A)=\{f \in X(A): \varrho[\lambda f]<+\infty \text { for some } \lambda>0\},
$$

see for example [27] and [4]. The subspace of $L^{\varrho}(A)$ defined by

$$
E^{\varrho}(A)=\left\{f \in L^{\varrho}(A): \varrho[\lambda f]<+\infty \text { for all } \lambda>0\right\}
$$


is called the space of the finite elements of $L^{\varrho}(A)$, see [27]. The following assumptions on modulars will be used

a) $\varrho$ is monotone, i.e. for $f, g \in X(A)$ and $|f| \leq|g|$ then $\varrho[f] \leq \varrho[g]$.

b) $\varrho$ is finite, i.e. denoting by $e_{0}$ the function $e_{0}(t)=1$ for every $t \in A, e_{0} \in L^{\varrho}(A)$. Note that clearly $e_{0} \in C_{u}(A)$.

c) $\varrho$ is absolutely finite, i.e. $\varrho$ is finite and for every $\varepsilon>0, \lambda>$ 0 there is $\delta>0$ such that $\varrho\left[\lambda \chi_{B}\right]<\varepsilon$ for any measurable subset $B \subset A$ with $\mu(B)<\delta$. Here $\chi_{B}$ denotes the characteristic function of the set $B$.

d) $\varrho$ is strongly finite, i.e. $e_{0} \in E^{\varrho}(A)$.

e) $\varrho$ is absolutely continuous, i.e. there exists $\alpha>0$ such that for every $f \in X(A)$, with $\varrho[f]<+\infty$, the following condition is satisfied: for every $\varepsilon>0$ there is $\delta>0$ such that $\varrho\left[\alpha f \chi_{B}\right]<\varepsilon$, for every measurable subset $B \subset A$ with $\mu(B)<\delta$.

For the above notions see, [27], [28] and [4]. Note that, since $\mu(A)<$ $+\infty$, if $\varrho$ is strongly finite and absolutely continuous then it is also absolutely finite (see [2]).

Classical examples of modular spaces are given by the Orlicz spaces generated by a $\varphi$-function $\varphi$ or, more generally, by any Musielak-Orlicz space generated by a $\varphi$-function $\varphi$ depending on a parameter, satisfying some growth condition with respect to the parameter (see [27], [23], [4] in some special cases). The modular functionals generating the above spaces satisfy all the previous assumptions.

We say that a sequence of functions $\left(f_{n}\right)_{n \in \mathbb{N}} \subset L^{\varrho}(A)$ is modularly convergent to a function $f \in L^{\varrho}(A)$, if there exists $\lambda>0$ such that

$$
\lim _{n \rightarrow+\infty} \varrho\left[\lambda\left(f_{n}-f\right)\right]=0 .
$$

This notion extends the norm-convergence in $L^{p}$-spaces. Moreover it is weaker than the F-norm-convergence induced by the Luxemburg F-norm generated by $\varrho$ and defined by

$$
\|f\|_{\rho} \equiv \inf \{u>0: \varrho[f / u] \leq u\} .
$$

We recall that a sequence of functions $\left(f_{n}\right)_{n \in \mathbb{N}}$ is F-norm-convergent (or strongly convergent) to $f$ iff

$$
\lim _{n \rightarrow+\infty} \varrho\left[\lambda\left(f_{n}-f\right)\right]=0
$$

for every $\lambda>0$. The two notions of convergence are equivalent if and only if the modular satisfies a $\Delta_{2}$-condition, i.e. there exists a constant $M>0$ such that $\varrho[2 f] \leq M \varrho[f]$, for every $f \in X(A)$, see [27]. For 
example, this happens for every $L^{p}$-spaces and Orlicz spaces generated by $\varphi$-functions with the $\Delta_{2}$-regularity condition (see [27], [4]). The modular convergence induces a topology on $L^{\varrho}(A)$, called modular topology. Given a subset $\mathcal{B} \subset L^{\varrho}(A)$, we will denote by $\overline{\mathcal{B}}$ the closure of $\mathcal{B}$ with respect to the modular topology. Then $f \in \overline{\mathcal{B}}$ if there is a sequence $\left(f_{n}\right)_{n \in \mathbb{N}} \subset \mathcal{B}$ such that $f_{n}$ is modularly convergent to $f$. Let us remark that $C(A) \subset L^{\varrho}(A)$ whenever $\varrho$ is monotone and finite. Indeed, for $\lambda>0$ we have $\varrho[\lambda f] \leq \varrho\left[\lambda\|f\|_{\infty} e_{0}\right]$, and so, since $e_{0} \in L^{\varrho}(A)$, we have $\lim _{\lambda \rightarrow 0^{+}} \varrho[\lambda f]=0$, that is $f \in L^{\varrho}(A)$. Analogously, if $\varrho$ is monotone and strongly finite, then $C(A) \subset E^{\varrho}(A)$.

We have the following (see [25] and [4]).

Proposition 1. Let $\varrho$ be a monotone, absolutely finite and absolutely continuous modular on $X(A)$. Then $\overline{C_{u}(A)}=L^{\varrho}(A)$.

\section{A Korovkin theorem in modular function spaces}

Let $e_{1}, \ldots e_{m}$ be $m$ functions in $C_{u}(A)$ such that the following property (P) holds: there exist continuous functions $a_{i} \in C_{u}(A), i=1, \ldots m$ such that the function

$$
P_{s}(t)=\sum_{i=1}^{m} a_{i}(s) e_{i}(t), s, t \in \bar{A}
$$

is positive and equal to zero if and only if $s=t$.

Let $\mathbf{T}=\left(T_{n}\right)_{n \in \mathbb{N}}$ be a family of positive linear operators $T_{n}: \mathcal{D} \rightarrow X(A)$, where $C_{u}(A) \subset \mathcal{D} \subset X(A)$. Here $\mathcal{D}$ is the domain of the operators $T_{n}$. We will assume that the family $\left(T_{n}\right)_{n \in \mathbb{N}}$ satisfies the following property

$(*)$ : there exists a subset $X_{\mathbf{T}} \subset \mathcal{D} \cap L^{\varrho}(A)$ with $C_{u}(A) \subset X_{\mathbf{T}}$ and a constant $R>0$ such that for every function $f \in X_{\mathbf{T}}$ we have $T_{n} f \in$ $L^{\varrho}(A)$ and

$$
\limsup _{n \rightarrow+\infty} \varrho\left[\lambda\left(T_{n} f\right)\right] \leq R \varrho[\lambda f]
$$

for every $\lambda>0$.

Note that if $T_{n}: \mathcal{D} \rightarrow X(A)$ are equi-continuous operators in $L^{\varrho}(A)$, i.e. $\varrho\left[\lambda T_{n} f\right] \leq R \varrho[\lambda f]$ for an absolute constant $R>0$ for every $\lambda>0$ and for every $f \in \mathcal{D} \cap L^{\varrho}(A)$, then clearly we can take $X_{\mathbf{T}}=L^{\varrho}(A) \cap \mathcal{D}$. We will provide an example of $T_{n}$ for which property $(*)$ holds for a suitable subspace $X_{\mathbf{T}} \neq L^{\varrho}(A) \cap \mathcal{D}$ but it is not continuous in $L^{\varrho}(A)$. 
110 Korovkin theorem in multivariate modular function spaces

In what follows, we will assume that

$$
\lim _{n \rightarrow+\infty} T_{n} e_{i}=e_{i}, \quad i=1, \ldots m \text { modularly in } L^{\varrho}(A) .
$$

Lemma 1. Let $\varrho$ be a monotone modular. Let the assumption (2) be satisfied and let us consider the function

$$
P(t)=\sum_{i=1}^{m} a_{i} e_{i}(t), t \in \bar{A}
$$

where $a_{i}$ are constants. Then $\lim _{n \rightarrow+\infty} T_{n} P=P$ modularly in $L^{\varrho}(A)$.

Proof. From (2) we can find a constant $\lambda>0$ such that

$$
\lim _{n \rightarrow+\infty} \varrho\left[\lambda\left(T_{n} e_{i}-e_{i}\right)\right]=0, i=1, \ldots m
$$

Let $M$ be such that $\left|a_{i}\right| \leq M$ for every $i=1, \ldots m$ and let $\alpha>0$ be such that $\alpha m M \leq \lambda$. Then, using the property of the modular, we get

$$
\varrho\left[\alpha\left(T_{n} P-P\right)\right] \leq \sum_{i=1}^{m} \varrho\left[\alpha m M\left(T_{n} e_{i}-e_{i}\right)\right] \leq \sum_{i=1}^{m} \varrho\left[\lambda\left(T_{n} e_{i}-e_{i}\right)\right]
$$

and so the assertion follows.

Lemma 2. Let $\varrho$ be a monotone modular. Let the assumptions (P) and (2) be satisfied. Then for the function $P_{s}(t)$ in (1) there holds $\lim _{n \rightarrow+\infty}\left(T_{n} P_{(\cdot)}\right)(\cdot)=0$ modularly in $L^{\varrho}(A)$.

Proof. Let $M>0$ be so large that $\left|a_{i}(s)\right| \leq M$ for every $i=1, \ldots, m$ and for every $s \in A$. From (2) we can find a constant $\lambda>0$ such that

$$
\lim _{n \rightarrow+\infty} \varrho\left[\lambda\left(T_{n} e_{i}-e_{i}\right)\right]=0, i=1, \ldots m
$$

Let $\alpha>0$ be such that $\alpha m M \leq \lambda$. Then

$$
\begin{aligned}
\varrho\left[\alpha\left(T_{n} P_{(\cdot)}\right)(\cdot)\right] & =\varrho\left[\alpha\left(\left(T_{n} P_{(\cdot)}\right)(\cdot)-P_{(\cdot)}(\cdot)\right)\right] \\
& \leq \sum_{i=1}^{m} \varrho\left[\alpha m M\left(T_{n} e_{i}-e_{i}\right)\right] \leq \sum_{i=1}^{m} \varrho\left[\lambda\left(T_{n} e_{i}-e_{i}\right)\right]
\end{aligned}
$$

and so the assertion follows.

Lemma 3. Let $\varrho$ be a finite, monotone and $Q$-quasi-convex modular. Let the assumptions $(P)$ and (2) be satisfied. Let $f_{s} \in C_{u}(A), s \in \bar{A}$, be a family of functions such that $f_{s}(t)$ is a continuous function of $(t, s) \in$ 
$\bar{A} \times \bar{A}$ and $f_{s}(s)=0$ for every $s \in \bar{A}$. Then $\lim _{n \rightarrow+\infty}\left(T_{n} f_{(\cdot)}\right)(\cdot)=$ 0 modularly in $L^{\varrho}(A)$.

Proof. Firstly, note that there exists a function $\widetilde{P}$ of the form $\widetilde{P}(t)=$ $\sum_{i=1}^{m} a_{i} e_{i}(t)$, such that $\widetilde{P}(t)>0$ for all $t \in \bar{A}$. Indeed, given two points $s_{1} \neq s_{2}$ of $\bar{A}$ we can take $\widetilde{P}=P_{s_{1}}+P_{s_{2}}$. Let us consider the diagonal $B=\{(s, s): s \in \bar{A}\}$. For a given $0<\varepsilon<1$, each point of $B$ has an open neighbourhood $U$ in $\bar{A} \times \bar{A}$ for which $\left|f_{s}(t)\right|<\varepsilon$ for every $(t, s) \in U$. We put $G=\bigcup U$ and $F=(\bar{A} \times \bar{A}) \backslash G$. Then $F$ is compact. Let $\theta=\min _{(t, s) \in F} P_{s}(t)>0, \Theta=\max _{(t, s) \in F}\left|f_{s}(t)\right|$. Clearly for every $(t, s) \in \bar{A} \times \bar{A}$ we have $\left|f_{s}(t)\right| \leq \varepsilon+\frac{\Theta}{\theta} P_{s}(t)$. Applying the operators $T_{n}$ we have

$$
\left|\left(T_{n} f_{s}\right)(s)\right| \leq \varepsilon\left(T_{n} e_{0}\right)(s)+\frac{\Theta}{\theta}\left(T_{n} P_{s}\right)(s) .
$$

Then, for $\gamma>0$ we have

$$
\begin{aligned}
\varrho\left[\gamma\left(T_{n} f_{(\cdot)}\right)(\cdot)\right] & \leq \varrho\left[2 \gamma \varepsilon\left(T_{n} e_{0}\right)(\cdot)\right]+\varrho\left[2 \gamma \frac{\Theta}{\theta}\left(T_{n} P_{(\cdot)}\right)(\cdot)\right] \\
& \leq Q \varepsilon \varrho\left[2 \gamma Q\left(T_{n} e_{0}\right)(\cdot)\right]+\varrho\left[2 \gamma \frac{\Theta}{\theta}\left(T_{n} P_{(\cdot)}\right)(\cdot)\right]=I_{1}+I_{2}
\end{aligned}
$$

Let us consider $I_{1}$. We can choose a positive constant $a>0$ such that $1=e_{0}(t) \leq a \widetilde{P}(t), t \in \bar{A}$. So applying the modular we have

$$
\begin{aligned}
\varrho\left[2 \gamma Q\left(T_{n} e_{0}\right)(\cdot)\right] & \leq \varrho\left[2 \gamma Q a\left(T_{n} \widetilde{P}\right)(\cdot)\right] \\
& \leq \varrho\left[4 \gamma Q a\left(\left(T_{n} \widetilde{P}\right)(\cdot)-\widetilde{P}(\cdot)\right)\right]+\varrho[4 \gamma Q a \widetilde{P}(\cdot)] \\
& =I_{1,1}+I_{1,2} .
\end{aligned}
$$

Let us consider $I_{1,1}$. By Lemma 1 , there exists $\alpha>0$ such that

$$
\varrho\left[\alpha\left(\left(T_{n} \widetilde{P}\right)(\cdot)-\widetilde{P}(\cdot)\right)\right]<1
$$

for sufficiently large $n$. For $I_{1,2}$ since the functions $e_{1}, \ldots, e_{m} \in$ $L^{\varrho}(A)$, there exists $\nu>0$ such that $\varrho\left[\nu e_{i}\right]<+\infty$ for every $i=$ $1, \ldots, m$. Now, putting $M=\max _{i=1, \ldots m}\left|a_{i}\right|$ and taking $\gamma$ such that $4 \gamma \operatorname{Qam} M<\nu$ and $4 \gamma Q a<\alpha$, we have

$$
I_{1,2}=\varrho[4 \gamma Q a \widetilde{P}]=\varrho\left[4 \gamma Q a \sum_{i=1}^{m} a_{i} e_{i}\right] \leq \sum_{i=1}^{m} \varrho\left[4 \gamma Q a m M e_{i}\right] \leq \sum_{i=1}^{m} \varrho\left[\nu e_{i}\right] .
$$

Thus we get $I_{1} \leq \varepsilon W$, for an absolute constant $W>0$. For $I_{2}$, by Lemma 2 , we can take $\gamma$ such that $\lim _{n \rightarrow+\infty} I_{2}=0$ modularly. Thus, for sufficiently small $\gamma>0$ we get $\lim _{n \rightarrow+\infty} \varrho\left[\gamma\left(\left(T_{n} f_{(\cdot)}\right)(\cdot)\right)\right]=0$. 
112 Korovkin theorem in multivariate modular function spaces

Lemma 4. Let $\varrho$ be a finite, monotone and $Q$-quasi-convex modular. Let the assumptions $(P)$ and $(2)$ be satisfied. Then for every $f \in C_{u}(A)$ we have

$$
\lim _{n \rightarrow+\infty} T_{n} f=f \quad \text { modularly in } L^{\varrho}(A) .
$$

Proof. Let $f \in C_{u}(A)$ be fixed. Let us take

$$
f_{s}(t)=f(t)-\frac{f(s)}{\widetilde{P}(s)} \widetilde{P}(t),
$$

where the function $\widetilde{P}$ is strictly positive in $\bar{A}$. By Lemma 3 there exists $\gamma>0$ such that $\lim _{n \rightarrow+\infty} \varrho\left[\gamma\left(T_{n} f_{(\cdot)}\right)(\cdot)\right]=0$ and this means that

$$
\lim _{n \rightarrow+\infty} \varrho\left[\gamma\left(\left(T_{n} f\right)(\cdot)-\frac{f(\cdot)}{\widetilde{P}(\cdot)}\left(T_{n} \widetilde{P}\right)(\cdot)\right)\right]=0 .
$$

For a constant $\delta>0$ we have

$$
\begin{aligned}
& \varrho\left[\delta\left(T_{n} f-f\right)\right] \leq \\
& \quad \leq \varrho\left[2 \delta\left(\left(T_{n} f\right)(\cdot)-\frac{f(\cdot)}{\widetilde{P}(\cdot)}\left(T_{n} \widetilde{P}\right)(\cdot)\right)\right]+\varrho\left[2 \delta\left(\frac{f(\cdot)}{\widetilde{P}(\cdot)}\left(T_{n} \widetilde{P}\right)(\cdot)-f(\cdot)\right)\right] \\
& \quad=\varrho\left[2 \delta\left(\left(T_{n} f\right)(\cdot)-\frac{f(\cdot)}{\widetilde{P}(\cdot)}\left(T_{n} \widetilde{P}\right)(\cdot)\right)\right]+\varrho\left[2 \delta \frac{f(\cdot)}{\widetilde{P}(\cdot)}\left(\left(T_{n} \widetilde{P}\right)(\cdot)-\widetilde{P}(\cdot)\right)\right] \\
& \quad=J_{1}+J_{2} .
\end{aligned}
$$

For $J_{1}$, if $2 \delta<\gamma$ we have $\lim _{n \rightarrow+\infty} J_{1}=0$. Moreover let $\Gamma:=$ $\max _{s \in \bar{A}}\left|\frac{f(s)}{\widetilde{P}(s)}\right|$, then $J_{2} \leq \varrho\left[2 \delta \Gamma\left(T_{n} \widetilde{P}-\widetilde{P}\right)\right]$ and so for sufficiently small $\delta>0$ we get $\lim _{n \rightarrow+\infty} J_{2}=0$ and so the assertion follows.

Remark 1. We remark that if assumption (2) holds in strong sense in $L^{\varrho}(A)$ then using exactly the same proof as before we can show that $\lim _{n \rightarrow+\infty} T_{n} f=f$ strongly in $L^{\varrho}(A)$ for every $f \in C_{u}(A)$.

The main theorem of this section is the following

Theorem 1. Let $\varrho$ be a monotone, absolutely finite, absolutely continuous and $Q$-quasi-convex modular on $X(A)$. Let $\mathbf{T}=\left(T_{n}\right)_{n \in \mathbb{N}}$ be a sequence of positive linear operators satisfying property $(*)$. Let the assumption $(P)$ be satisfied. Then if

$$
\lim _{n \rightarrow+\infty} T_{n} e_{i}=e_{i} i=1, \ldots, m, \text { strongly in } L^{\varrho}(A)
$$

then $\lim _{n \rightarrow+\infty} T_{n} f=f, \quad$ modularly in $L^{\varrho}(A)$ for each $f \in L^{\varrho}(A) \cap \mathcal{D}$ such that $f-C_{u}(A) \subset X_{\mathbf{T}}$. 
Proof. Let $f \in L^{\varrho}(A) \cap \mathcal{D}$ be a function such that $f-C_{u}(A) \subset X_{\mathbf{T}}$. By Proposition 1, there is a $\lambda>0$ and a sequence $\left(f_{k}\right)_{k \in N} \subset C_{u}(A)$ such that $\varrho[3 \lambda f]<+\infty$ and $\lim _{n \rightarrow+\infty} \varrho\left[3 \lambda\left(f_{k}-f\right)\right]=0$. Let $\varepsilon>0$ be fixed and let $\bar{k}$ be such that for every $k \geq \bar{k}, \varrho\left[3 \lambda\left(f_{k}-f\right)\right]<\varepsilon$. Fix now $\bar{k}$, then we have

$$
\varrho\left[\lambda\left(T_{n} f-f\right)\right] \leq \varrho\left[3 \lambda T_{n}\left(f-f_{\bar{k}}\right)\right]+\varrho\left[3 \lambda\left(T_{n} f_{\bar{k}}-f_{\bar{k}}\right)\right]+\varrho\left[3 \lambda\left(f_{\bar{k}}-f\right)\right] .
$$

Passing to limsup, taking into account Remark 1 and property $(*)$, we obtain $\lim \sup _{n \rightarrow+\infty} \varrho\left[\lambda\left(T_{n} f-f\right)\right] \leq \varepsilon(R+1)$ and the assertion follows from the arbitrariness of $\varepsilon>0$.

Remark 2. Note that a similar result holds true in the case when $A$ is compact replacing of course $C_{u}(A)$ with $C(A)$. The proof is exactly the same.

\section{Application to discrete operators}

Let $A \subset \mathbb{R}^{N}$ be a bounded open set and let $(r(n))_{n \in \mathbb{N}}$ be an increasing sequence of natural numbers.

For every fixed $n \in \mathbb{N}$, by $\Gamma_{n}=\left(\nu_{n, k}\right)_{k=0,1, \ldots, r(n)} \subset A, \nu_{n, k}=$ $\left(\nu_{n, k}^{1}, \ldots, \nu_{n, k}^{N}\right)$, we denote a finite sequence of points such that $\overline{U \Gamma_{n}}=A$. Let us consider a sequence $\mathbf{S}=\left(S_{n}\right)_{n \in \mathbb{N}}$ of positive operators of the form

$$
\left(S_{n} f\right)(s)=\sum_{k=0}^{r(n)} K_{n}\left(s, \nu_{n, k}\right) f\left(\nu_{n, k}\right), n \in \mathbb{N}, s \in A
$$

where $\left(K_{n}\right)_{n \in \mathbb{N}}, K_{n}: A \times \Gamma_{n} \rightarrow \mathbb{R}$ is a sequence of nonnegative measurable functions such that

$$
\sum_{k=0}^{r(n)} K_{n}\left(s, \nu_{n, k}\right)=1 \text { for every } n \in \mathbb{N}, s \in A .
$$

Note that the domain of the operator (3) contains the space $X(A)$, due to the nature of the operator. Here $X(A)$ is the space of all real valued measurable functions which are everywhere defined on $A$ (i.e. we distinguish two equivalent but different functions).

For every $j=1, \ldots, N$ and $s=\left(s_{1}, \ldots, s_{N}\right)$ we put

$$
m_{j}\left(K_{n}, s\right):=\sum_{k=0}^{r(n)} K_{n}\left(s, \nu_{n, k}\right)\left(\nu_{n, k}^{j}-s_{j}\right), M_{2}\left(K_{n}, s\right):=\sum_{k=0}^{r(n)} K_{n}\left(s, \nu_{n, k}\right)\left|\nu_{n, k}-s\right|^{2} .
$$


We put $e_{0}(t)=1, e_{i}(t)=t_{i}$ for $i=1, \ldots, N$ and $e_{N+1}(t)=|t|^{2}, t=$ $\left(t_{1}, \ldots, t_{N}\right) \in A$. Note that these functions satisfy property (1) taking

$$
P_{s}(t)=|t-s|^{2},(t, s) \in \bar{A} \times \bar{A} .
$$

According to the above assumptions we have immediately $S_{n} e_{0}=e_{0}=$ 1 , for every $n \in \mathbb{N}$. We have the following

Proposition 2. Let $\varrho$ be a finite and monotone modular on $X(A)$. Then a necessary and sufficient condition that

$$
\lim _{n \rightarrow+\infty} m_{j}\left(K_{n}, \cdot\right)=0, j=1, \ldots, N, \lim _{n \rightarrow+\infty} M_{2}\left(K_{n}, \cdot\right)=0
$$

modularly (strongly) in $L^{\varrho}(A)$ is that $\lim _{n \rightarrow+\infty} S_{n} e_{j}=e_{j}, j=1, \ldots, N+1$, modularly (strongly) in $L^{\varrho}(A)$.

Proof. We prove the proposition in case of strongly convergence. We can assume $\lambda=1$. First we prove the necessary condition. It is obvious that $\left(S_{n} e_{j}\right)(s)-e_{j}(s)=m_{j}\left(K_{n}, s\right), j=1, \ldots, N$. Moreover

$$
\left(S_{n} e_{N+1}\right)(s)-e_{N+1}(s)=M_{2}\left(K_{n}, s\right)+2 \sum_{j=1}^{N} e_{j}(s) m_{j}\left(K_{n}, s\right) .
$$

Passing to the modular we have $\varrho\left[S_{n} e_{j}-e_{j}\right]=\varrho\left[m_{j}\left(K_{n}, \cdot\right)\right], \quad j=$ $1, \ldots, N$ and

$$
\varrho\left[S_{n} e_{N+1}-e_{N+1}\right] \leq \varrho\left[2 M_{2}\left(K_{n}, \cdot\right)\right]+\sum_{j=1}^{N} \varrho\left[4 N\left\|e_{j}\right\|_{\infty} m_{j}\left(K_{n}, \cdot\right)\right]
$$

that is the assertion. For the sufficient condition, note that

$$
M_{2}\left(K_{n}, s\right)=\left(S_{n} e_{N+1}\right)(s)-e_{N+1}(s)-2 \sum_{j=1}^{N} e_{j}(s)\left(\left(S_{n} e_{j}\right)(s)-e_{j}(s)\right)
$$

and so applying the modular, as before, we obtain the assertion.

We have the following corollary

Corollary 1. Let $\varrho$ be a monotone, strongly finite, absolutely continuous and $Q$-quasi-convex modular on $X(A)$. Assume that the family $\left(S_{n}\right)_{n \in \mathbb{N}}$ satisfies property $(*)$ and $(4)$ holds in the strong sense. Then $\lim _{n \rightarrow+\infty} S_{n} f=f$, modularly in $L^{\varrho}(A)$ for each $f \in L^{\varrho}(A)$ such that $f-C_{u}(A) \subset X_{\mathbf{S}}$, where $X_{\mathbf{S}}$ is the corresponding class given in property $(*)$. 
Here we will describe the class $X_{\mathbf{S}}$ in some particular case. Let $\Phi$ be the class of all functions $\varphi: \mathbb{R}_{0}^{+} \rightarrow \mathbb{R}_{0}^{+}$such that $\varphi$ is a convex function, $\varphi(0)=0, \varphi(u)>0$ for $u>0$ and $\lim _{u \rightarrow+\infty} \varphi(u)=+\infty$.

For $\varphi \in \Phi$, we define for every $f \in X(A)$, the functional

$$
\varrho^{\varphi}[f]=\int_{A} \varphi(|f(s)|) d s .
$$

As it is well known, $\varrho^{\varphi}$ is a convex modular on $X(A)$ and the subspace

$$
L^{\varphi}(A)=\left\{f \in X(A): \varrho^{\varphi}[\lambda f]<+\infty \text { for some } \lambda>0\right\}
$$

is the Orlicz space generated by $\varphi$, (see [27]). The subspace of $L^{\varphi}(A)$, defined by

$$
E^{\varphi}(A)=\left\{f \in X(A): \varrho^{\varphi}[\lambda f]<+\infty \text { for every } \lambda>0\right\},
$$

is called the space of finite elements of $L^{\varphi}(A)$. For example every bounded function belongs to $E^{\varphi}(A)$. Note that this modular satisfies all the assumptions listed in Section 2.

Let us consider the sequence of operator (3) and let us assume that

$$
\int_{A} K_{n}\left(s, \nu_{n, k}\right) d s \leq \xi_{n}
$$

where $\xi_{n}$ is a bounded sequence of positive numbers. For every $n \in \mathbb{N}$, we define

$$
\varrho_{n}^{\varphi}[f]=\sum_{k=0}^{r(n)} \varphi\left(\left|f\left(\nu_{n, k}\right)\right|\right), f \in X(A) .
$$

Now, let us denote by $\mathcal{A}_{\varphi}$ the class of all functions in $L^{\varphi}(A)$ such that

$$
\limsup _{n \rightarrow+\infty} \xi_{n} \varrho_{n}^{\varphi}[\lambda f] \leq R \varrho^{\varphi}[\lambda f]
$$

for every $\lambda>0$ and an absolute constant $R>0$ independent of $f$ and $\lambda$. We have the following

Proposition 3. $\mathcal{A}_{\varphi} \subset X_{\mathbf{S}}$.

Proof. Let $\lambda>0$ be fixed. Using the Jensen inequality and the assumptions on the kernel $\left(K_{n}\right)_{n \in \mathbb{N}}$, we get

$$
\varrho^{\varphi}\left[\lambda S_{n} f\right] \leq \xi_{n} \sum_{k=0}^{r(n)} \varphi\left(\lambda\left|f\left(\nu_{n, k}\right)\right|\right)=\xi_{n} \varrho_{n}^{\varphi}[\lambda f]
$$


and so, passing to the limsup, we obtain immediately

$$
\limsup _{n \rightarrow+\infty} \varrho^{\varphi}\left[\lambda S_{n} f\right] \leq R \varrho^{\varphi}[\lambda f]
$$

Example 1. Let $A=[0,1]^{N}$. Let $n \in \mathbb{N}$ be fixed and let $r_{i}(n)$, a finite sequence of positive integers, $i=1, \ldots N$. Let us consider a multi-index $h=$ $\left(h_{1}, \ldots, h_{N}\right) \in \mathbb{N}^{N}$, such that $0 \leq h_{i} \leq r_{i}(n)$, for every $i=1, \ldots, N$. For any choice of $h$ we consider the vector $\nu_{n, h}=\left(\nu_{n, h_{1}}^{1}, \ldots, \nu_{n, h_{N}}^{N}\right)$, where for every $i=1, \ldots, N,\left(\nu_{n, h_{i}}^{i}\right), i=0, \ldots r_{i}(n)$, is a finite partition of the interval $I=[0,1]$ of the i-axis. Putting

$$
\Delta_{h}^{n}:=\prod_{i=1}^{N}\left(\nu_{n, h_{i}}^{i}-\nu_{n, h_{i}-1}^{i}\right),
$$

let us assume that there exist two sequences $\left(a_{n}\right),\left(b_{n}\right)$ of positive real numbers, such that $0<a_{n} \leq \Delta_{h}^{n} \leq b_{n}$, for every $h \in \mathbb{N}^{N}$ and $n \in \mathbb{N}$, and $b_{n} \rightarrow 0, n \rightarrow+\infty$. By a renumbering of the vectors $\nu_{n, h}$ into a sequence $\widetilde{\nu}_{n, k}, \quad k=0,1, \ldots \widetilde{r}(n)$, let us consider a kernel $K_{n}\left(s, \widetilde{\nu}_{n, k}\right)$, satisfying the above assumptions and let $\xi_{n}$ be the corresponding sequences of numbers which dominate the integrals over $A$. Finally, let us assume that $0 \leq \xi_{n} / a_{n} \leq M$, for a fixed constant $M>0$ and any $n \in \mathbb{N}$. Thus, in this instance, the class $\mathcal{A}_{\varphi}$ contains all the Riemann integrable functions over $A$. Indeed, we have, for $\lambda=1$

$$
\begin{aligned}
\limsup _{n \rightarrow+\infty} \xi_{n} \sum_{h_{1}=0}^{r_{1}(n)} & \cdots \sum_{h_{N}=0}^{r_{N}(n)} \varphi\left(\left|f\left(\nu_{n, h}\right)\right|\right) \\
& \leq \limsup _{n \rightarrow+\infty} \frac{\xi_{n}}{a_{n}} \sum_{h_{1}=0}^{r_{1}(n)} \cdots \sum_{h_{N}=0}^{r_{N}(n)} \varphi\left(\left|f\left(\nu_{n, h}\right)\right|\right) \Delta_{h}^{n} \\
& \leq M \limsup _{n \rightarrow+\infty} \sum_{h_{1}=0}^{r_{1}(n)} \cdots \sum_{h_{N}=0}^{r_{N}(n)} \varphi\left(\left|f\left(\nu_{n, h}\right)\right|\right) \Delta_{h}^{n} .
\end{aligned}
$$

The last sum is a Riemann sum of the function $\varphi \circ|f|$ and so, if $f$ is Riemann integrable, then the above limsup is dominated by the integral $M \int_{A} \varphi(|f(s)|) d s$ and from this the assertion follows. 


\section{Application to Mellin-type operators}

Let us consider $A=[0,1]^{N}$ and for any vectors $t=\left(t_{1}, \ldots, t_{N}\right), s=$ $\left(s_{1}, \ldots, s_{N}\right) \in A$, we put $t s=\left(t_{1} s_{1}, \ldots, t_{N} s_{N}\right)$. Let $\left(K_{n}\right)_{n \in \mathbb{N}}$ be a sequence of kernel functions $K_{n}: A \rightarrow \mathbb{R}_{0}^{+}$such that

$$
\int_{A} K_{n}(t) d t=1 \text { and } \int_{A} \frac{K_{n}(t)}{t_{1} \cdots t_{N}} d t \leq W
$$

for every $n \in \mathbb{N}$ and $W$ is an absolute constant. Here, for a sake of simplicity, we consider an Orlicz space. Let $\varphi \in \Phi$ be fixed and let $L^{\varphi}(A)$ be the corresponding Orlicz space. For any function $f \in L^{\varphi}(A)$ we define the positive linear operator

$$
\left(T_{n} f\right)(s)=\int_{A} K_{n}(t) f(t s) d t, s \in A .
$$

In this instance we can show that $L^{\varphi}(A) \subset \mathcal{D}=\operatorname{Dom} \mathbf{T}=\bigcap_{n \in \mathbb{N}} D o m T_{n}$, where $\operatorname{Dom}_{n}$ is the subset of $X(A)$ on which $T_{n} f$ is well defined as a measurable function of $s \in A$. A first result on these operators is given by the following proposition.

Proposition 4. $T_{n} f \in L^{\varphi}(A)$ whenever $f \in L^{\varphi}(A)$ and

$$
\varrho^{\varphi}\left[T_{n} f\right] \leq W \varrho^{\varphi}[f] .
$$

Proof. By the Jensen inequality and the Fubini-Tonelli theorem, we have

$$
\begin{aligned}
\varrho^{\varphi}\left[T_{n} f\right] & \leq \int_{A} K_{n}(t)\left[\int_{A} \varphi(|f(t s)|) d s\right] d t \\
& \leq \int_{A} \frac{K_{n}(t)}{t_{1} \ldots t_{N}} \varrho^{\varphi}[f] d t \leq W \varrho^{\varphi}[f] .
\end{aligned}
$$

As a consequence of the above proposition we get $X_{\mathbf{T}}=L^{\varphi}(A)$. We define the integral moments $m_{i}\left(K_{n}, s\right)$ and $m_{i, 2}\left(K_{n}, s\right)$ on putting, for $i=1, \ldots, N$,

$$
m_{i}\left(K_{n}, s\right)=s_{i} \int_{A} K_{n}(t)\left(t_{i}-1\right) d t, m_{i, 2}\left(K_{n}, s\right)=s_{i}^{2} \int_{A} K_{n}(t)\left(t_{i}-1\right)^{2} d t .
$$

As in discrete case, according to the above assumptions, we have immediately $T_{n} e_{0}=e_{0}=1$ for every $n \in \mathbb{N}$. Moreover we have 
Proposition 5. A necessary and sufficient condition that

$$
\lim _{n \rightarrow+\infty} m_{i}\left(K_{n}, \cdot\right)=0, \text { and } \lim _{n \rightarrow+\infty} m_{i, 2}\left(K_{n}, \cdot\right)=0, i=1, \ldots, N,
$$

modularly (strongly) in $L^{\varphi}(A)$ is that

$$
\lim _{n \rightarrow+\infty} T_{n} e_{i}=e_{i}, \text { and } \lim _{n \rightarrow+\infty} T_{n} e_{i}^{2}=e_{i}^{2}, i=1, \ldots, N,
$$

modularly (strongly) in $L^{\varphi}(A)$, where $e_{i}(t)=t_{i}, i=1, \ldots, N$.

Proof. The proof follows from the identities, $m_{i}\left(K_{n}, s\right)=\left(T_{n} e_{i}-e_{i}\right)(s)$ and $m_{i, 2}\left(K_{n}, s\right)=\left(T_{n} e_{i}^{2}-e_{i}^{2}\right)(s)-2 s_{i} m_{i}\left(K_{n}, s\right)$, for $i=1, \ldots, N$.

As a consequence we get the following corollary

Corollary 2. If the moments $m_{i}\left(K_{n}, \cdot\right)$ and $m_{i, 2}\left(K_{n}, \cdot\right), i=$ $1, \ldots, N$, are strongly convergent to zero then $\lim _{n \rightarrow+\infty} T_{n} f=f$, modularly in $L^{\varphi}(A)$ for each $f \in L^{\varphi}(A)$.

Proof. We only remark that, putting $e_{N+1}(t)=|t|^{2}$, we have also $\lim _{n \rightarrow+\infty} T_{n} e_{N+1}=e_{N+1}$ strongly in $L^{\varphi}(A)$.

Remark 3 Note that the above results hold also in abstract modular function spaces. In this instance, besides the above assumptions on the generating modular $\varrho$, (monotonicity, absolute finiteness and absolute continuity), we have to assume some generalized Jensen convexity, in integral form and a notion of subboundedness (see e.g. [4]). In particular we have to assume an inequality of the form $\varrho[f(t \cdot)] \leq F(t) \varrho[f(\cdot)]$ where $F$ is a measurable function such that $\int_{A} K_{n}(t) F(t) d t \leq W$ for every $n \in \mathbb{N}$ and an absolute constant $W>0$. These assumptions are automatically satisfied in Orlicz spaces and are fundamental in order to obtain the modular continuity of the operators $T_{n}$ (Proposition 4).

Aknowledgments. We wish to express our deep gratitude to the Referees for their valuable and interesting suggestions which improved the paper.

\section{References}

[1] F. Altomare and M. Campiti, Korovkin-Type Approximation Theory and its Applications, Walter de Gruyter, Berlin, New York, 1994.

[2] C. Bardaro and I. Mantellini, Approximation properties in abstract modular spaces for a class of general sampling type operators, Applicable Analysis, 85 (2006), 383-413.

[3] C. Bardaro and I. Mantellini, Korovkin theorem in modular spaces, Commentationes Math., 47, (2007), 239-253. 
[4] C. Bardaro, J. Musielak and G. Vinti, Nonlinear Integral Operators and Applications, De Gruyter Series in Nonlinear Analysis and Appl., Vol. 9, 2003.

[5] H. Berens and G.G. Lorentz, Theorems of Korovkin type for positive linear operators on Banach lattices, In: Approximation Theory (Proc. Internat. Sympos. Univ. Texas, Austin,Tex., (1973), 1-30; Academic Press, New York, 1973.

[6] S.N. Bernstein, Demonstration du theoreme de Weierstrass fondee sur le calcul de probabilities, Com. of the Kharkov Math. Soc., 13 (1912), $1-2$.

[7] H. Bohman, On approximation of continuous and of analytic functions, Arkiv Math., 2 (1952), 43-56.

[8] E. Briem, Convergence of sequences of positive operators on $L^{p}$-spaces, In: Proc. of the Nineteenth Nordic Congress of Mathematicians, Reykjavik, (1984), 126-131, Visindafel. Isl., XLIV, Icel. Math. Soc. Reykjavik, 1985.

[9] P.L. Butzer and R.J. Nessel, Fourier Analysis and Approximation I, Academic Press, New York, London, 1971.

[10] P.L. Butzer and S. Jansche, A direct approach to the Mellin tramsform, J. Fourier Anal. Appl., 3 (1997), 325-375.

[11] F. Degani Cattelani, Nuclei di tipo "distanza" che attutiscono i salti in una o piú variabili, Atti Sem. Mat. Fis. Univ. Modena, 30 (1981), $299-321$.

[12] R.A. DeVore, The Approximation of Continuous Functions by Positive Linear Operators, Lecture notes in Math., 293, Springer-Verlag, 1972.

[13] R.A. DeVore and G.G. Lorentz, Constructive Approximation, Grund. Math. Wiss. 303, Springer Verlag, 1993.

[14] K. Donner, Korovkin theorems in $L^{p}$-spaces, J. Funct. Anal., 42 (1981), $12-28$.

[15] K. Donner, Extension of Positive Operators and Korovkin Theorems, Lecture Notes in Math., 904, Springer-Verlag, Berlin, 1982.

[16] S.M. Eisenberg, Korovkin's theorem, Bull. Malaysian Math. Soc., 2 (1979), 13-29.

[17] C. Fiocchi, Two-dimensional moment kernels and convergence in area, Atti Sem. Mat. Fis. Univ. Modena, 33 (1986), 291-311.

[18] M.W. Grossman, Note on a generalized Bohman-Korovkin theorem, J. Math. Anal. Appl., 45 (1974), 43-46. 
120 Korovkin theorem in multivariate modular function spaces

[19] R.L. James, The extension and convergence of positive operators, J. Approx. Theory, 7 (1973), 186-197.

[20] W. Kitto and D.E. Wulbert, Korovkin approximations in $L^{p}$-spaces, Pacific J. Math., 63 (1976), 153-167.

[21] P.P. Korovkin, On convergence of linear positive operators in the spaces of continuous functions (Russian), Doklady Akad. Nauk. S.S.S.R., 90 (1953), 961-964.

[22] P.P. Korovkin, Linear Operators and Approximation Theory, Hindustan, Delhi, 1960.

[23] W.M. Kozlowski, Modular Function Spaces, Pure Appl. Math., Marcel Dekker, New York and Basel, 1988.

[24] G.G. Lorentz, Approximation of Functions, Chelsea Publ. Comp. New York, 1986.

[25] I. Mantellini, Generalized sampling operators in modular spaces, Commentationes Math., 38, (1998), 77-92.

[26] M.J. Marsden and S.D. Riemenschneider, Korovkin theorems for integral operators with kernels of finite oscillation, Canad. J. Math., 26 (1974), 1390-1404.

[27] J. Musielak, Orlicz Spaces and Modular Spaces, Springer-Verlag, Lecture Notes in Math., 1034 (1983).

[28] J. Musielak, Nonlinear approximation in some modular function spaces I, Math. Japon., 38, (1993), 83-90.

[29] H. Nakano, Modulared Semi-Ordered Linear Spaces, Maruzen Co., Ltd, Tokyo, 1950.

[30] P. Renaud, A Korovkin theorem for abstract Lebesgue spaces, J. Approx. Theory, 102, (2000), 13-20.

[31] E. Schäfer, Korovkin's theorems: a unifying version, Functiones et Approximatio, 18 (1989), 43-49.

[32] P.M. Soardi, On quantitative Korovkin's theorem in multivariate Orlicz spaces, Math. Japonica, 48 (1998), 205-212.

Department of Mathematics and Informatics

University of Perugia

Via Vanvitelli 1, 06123 Perugia

ITALY

(E-mail : bardaro@unipg.it)

(E-mail : mantell@dipmat.unipg.it)

(Received : April 2008) 


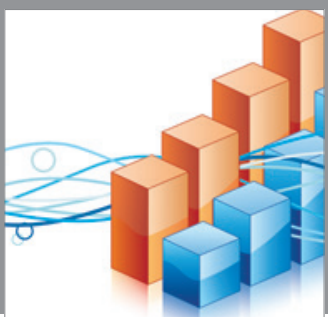

Advances in

Operations Research

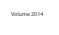

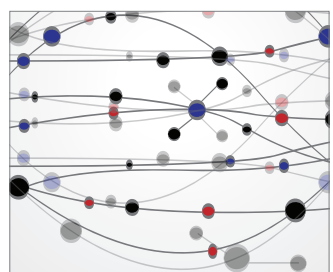

\section{The Scientific} World Journal
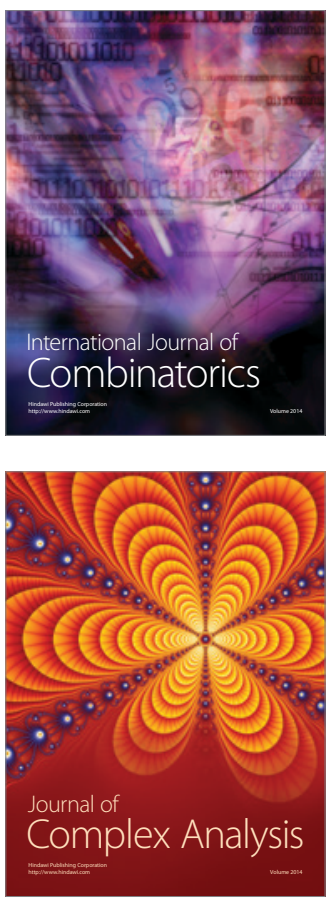

International Journal of

Mathematics and

Mathematical

Sciences
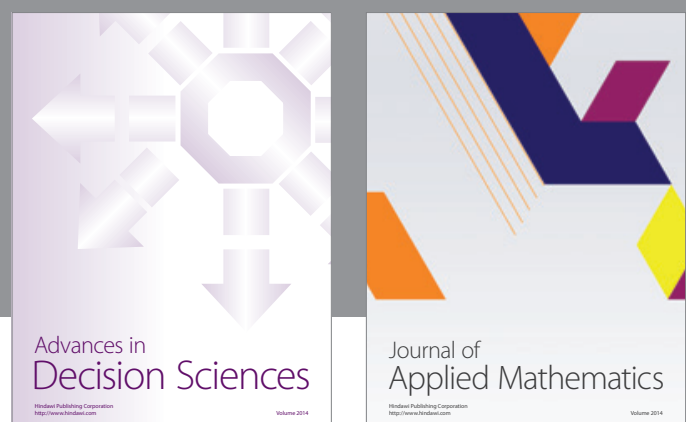

Journal of

Applied Mathematics
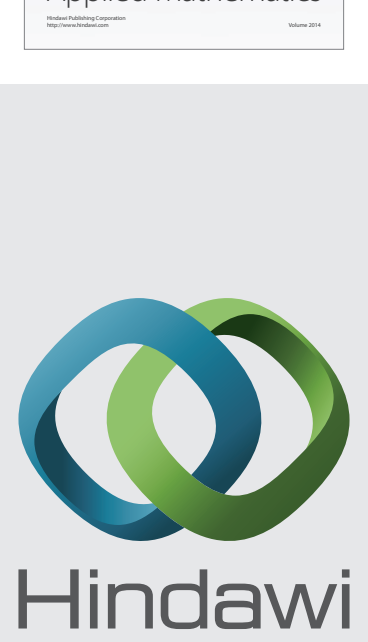

Submit your manuscripts at http://www.hindawi.com
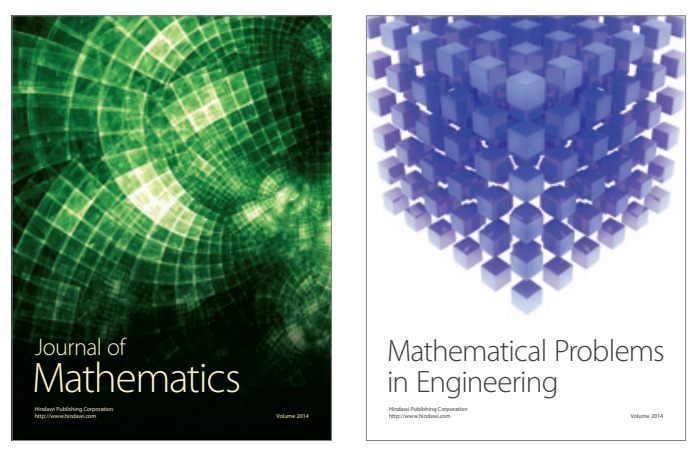

Mathematical Problems in Engineering
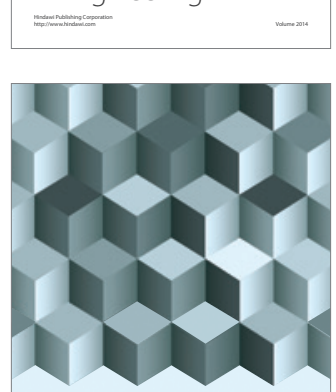

Journal of

Function Spaces
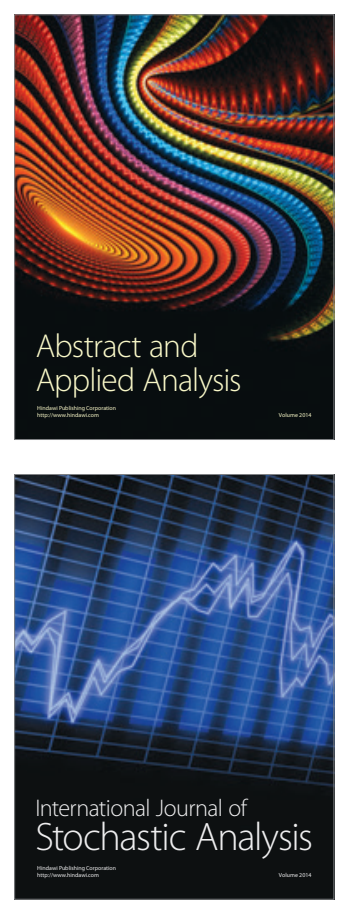

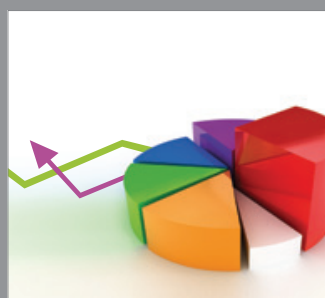

ournal of

Probability and Statistics

Promensencen
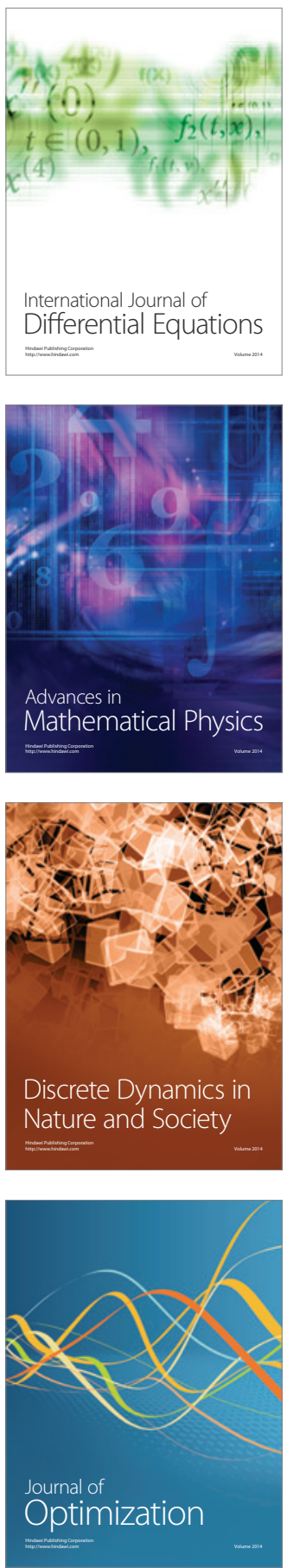